\title{
Tackling the drivers of child undernutrition in developing countries: what works and how should interventions be designed?
}

\author{
Pat Pridmore* and Roy Carr-Hill \\ Department of Education, Health and International Development, Institute of Education, University of London, \\ 20 Bedford Way, London WC1H OAL, UK
}

Submitted 24 August 2009: Accepted 13 May 2010: First published online 19 July 2010

\begin{abstract}
Objective: The present paper presents a synthesis of available evidence to support action on the interventions that can effectively address the main drivers of child undernutrition in developing countries. It also discusses how interventions should be designed and identifies policy-relevant areas for further research.

Design: A structured literature review of fifty-eight controlled evaluations and studies in developing countries were selected because they are methodologically sound, recent (reported within the past 10 years), report on nutritional status, cover a wide range of interventions and are implemented by a range of different agencies. Setting: Indirect interventions in developing countries, which address the underlying and basic causes of child undernutrition and can potentially be implemented in the short to medium term.

Subjects: Children under 5 years of age and their mothers across a range of developing countries.

Results: Evidence has now accumulated to guide action on a range of indirect interventions that can reduce child undernutrition, but for all these interventions context is all-important. There is less evidence on how these interventions can be effectively implemented on a large scale.

Conclusions: Efforts should be directed towards improving implementation of effective interventions on a large scale. Donors need to keep commitments both in dollar terms and in terms of the Paris Declaration so that the push for improved nutrition does not become donor driven. At the country level, there is a need for enabling governance structures, institutions and evidence-based decision making. Within countries there is a need for well-trained personnel with delegated authority, accountable to local people. It is essential for chains of accountability to be transparent and for active involvement of households in decision-making processes.
\end{abstract}

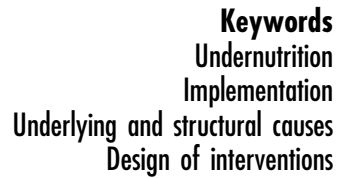

Keywords

Undernutrition

Design of interventions
Access to adequate food and nutrition is a basic human right and yet the global burden of child undernutrition remains substantial, being driven mainly by poverty, social inequities and lack of enough nutritious food. The problem needs to be addressed urgently because the food, fuel and financial crises, coming together on top of climate changes, are threatening the livelihoods of millions of people in developing countries. Governments also need to pay more attention to nutrition because it is a crucial input for achieving the Millennium Development Goals that respond to the world's main development challenges and because nutrition is now recognised to be a governance issue ${ }^{(1)}$.

The series of papers on Maternal and Child Undernutrition published in The Lancet in 2008 defined the extent of child undernutrition and showed that the consequences are long term, irreversible and impact human capital development. They also found that the problem is of low national priority in nearly all high-burden countries and that there are cost-effective, direct interventions (e.g. vitamin A and zinc supplementation for children and multiple micronutrient supplementation for pregnant women), but these are not being implemented on a large scale ${ }^{(2-5)}$.

However, these papers did not go far enough in addressing the underlying and basic causes of child undernutrition and there have since been calls for a review of the available evidence on how to address these causes $^{(6)}$. The present paper responds to these calls by reviewing evidence from a structured review of fifty-eight controlled evaluations and studies (surveys, reviews, etc.) of nutrition programmes that addressed child undernutrition indirectly and have been selected because they 
are methodologically sound, reported within the past 10 years, have reported on nutritional status and cover a wide range of interventions implemented by a range of different agencies. Methodological details and outcomes were extracted by the authors of the present paper who independently assessed the quality of the papers (full details of the search strategy, the criteria for selecting the studies and evaluations and the framework for analysis are available from the authors).

The present paper draws on the findings from the review of available evidence to discuss the constraints to implementing and assessing effective programmes at the macro (national and global), meso (local government/ district) and micro (individual/family/household) levels and to consider how these constraints can be addressed. Finally, the present paper identifies areas for further research and defines some questions that need to be explored.

\section{Evidence for the effectiveness of indirect interventions}

The conceptual framework for understanding the causes of child undernutrition, originally developed by $\mathrm{UNICEF}^{(7)}$, but now agreed to by most stakeholders, has been used to structure the analysis and presentation of the findings. This framework recognises that the causes of child undernutrition are complex, multiple and interactive. The underlying causes are lack of household access to and use of nutritious foods, health services, water and sanitation and poor child-care practices. The root causes are the structural barriers in society caused by the unfair institutional structures, political and ideological frameworks and economic structures that lead to social injustice and reduce women's status and autonomy. In the interests of brevity, not all possible interventions are dealt with extensively. Indeed, some of the more obvious ones such as increasing food availability nationally, advancing women's formal education, ensuring a clean environment and raising household incomes in real terms are not considered in depth, but see the seminal papers by Mosley and $\mathrm{Chen}^{\left({ }^{(8)}\right.}$ and by Caldwell $^{(9)}$ whose work has led to an established body of research in this field and the review by Hills ${ }^{(10)}$. We have not considered some of the more obvious interventions partly because they are obvious and partly because they can only be implemented on a large scale over a long period (see subsection on macro-economic policy reform). Instead, those seen by the authors as most innovative and potentially implementable on a large scale in the more medium term are highlighted.

\section{Increasing bousehold food production and nutrient consumption}

Ceteris paribus, raising household incomes either through employment or home-based production, and increasing food availability, can both increase nutrient consumption among children ${ }^{(6)}$. However, if the former is to include the whole population (rather than being targeted at specific groups), it is a very long-term programme, especially in the current economic climate, and the latter struggles to keep pace with growing populations. Alternatives that have been tried include biofortification and home gardening.

Biofortification programmes to increase the micronutrient density and bioavailability of staple food crops are still at the experimental stage with the HarvestPlus initiative playing a key role in research and development. Predictive analyses suggest that although biofortification could be one of the most cost-effective ways to provide micronutrients to families that are poor and do not have good access to health services ${ }^{(11,12)}$, it is imperative to integrate the biofortification approach into existing programmes and demonstrate the on-the-ground costeffectiveness of biofortification in comparison to alternative interventions ${ }^{(13)}$. There is evidence that home gardening interventions can also increase the diversity of children's diets and reduce micronutrient deficiencies ${ }^{(14)}$. These interventions have also led to the empowerment of women ${ }^{(15,16)}$ and been used to integrate agriculture and primary health activities ${ }^{(17)}$.

\section{Improving the quality of care given to young children}

More highly educated women usually provide better quality of care for their children, but this is confounded by income ${ }^{(18)}$. Moreover, although maternal education targeted towards improving child-care practices has not always been effective on its own ${ }^{(19)}$, a study in Madagascar showed that it can be effective where participatory approaches are used, networks of women's groups are supportive and the quality of the child-care services delivered is improved by training local community nutrition workers ${ }^{(20)}$. But the pathways that can translate maternal education into improved child nutrition need to be further clarified.

A randomised controlled trial of a multidisciplinary programme in Iran also showed that maternal education can be effective as part of broad-based multidisciplinary actions. The study showed that stunting could be significantly reduced by educating mothers about child care, growth and hygiene, strengthening women's literacy, promoting home gardening and consumption of healthy foods, establishing cooperative stores, improving opportunities for employment and income generation and providing inputs to improve water and sanitation ${ }^{(21)}$. These actions were implemented through non-governmental organisations (NGO).

\section{Increasing access to and appropriate use of adequate bealth services, water and sanitation} Ideally, of course, the health environment (including health services, water and sanitation) should be dramatically improved for all; but, once again, these are longterm goals and the concern here is with the possibility of 
more medium-term solutions, the most currently favoured being conditional cash transfers (CCT). A systematic review of CCT to disadvantaged families' conditions on carers using maternal and child health (MCH) services found that they significantly reduced stunting in Mexico and Nicaragua where carers had access to quality services ${ }^{(22)}$. The present review endorses the findings of other researchers ${ }^{(23,24)}$.

However, in Mexico, an analysis of census data, mortality registries, the nominal registry of children and national nutrition surveys found that these CCT programmes have been most successful as one in a series of cost-effective interventions implemented incrementally (together with universal child immunisation, clean water programmes and vitamin A distribution) as part of a comprehensive social and economic policy development strategy ${ }^{(25)}$.

\section{Strengthening governance to create an enabling environment and increasing community influence over decision making}

Evidence from a quantitative analysis of country-level data from eighty-two countries found that countries with higher scores on government effectiveness, political stability and rule of law have lower levels of child undernutrition $^{(26)}$. An in-depth case study of governance in Madagascar found that 'voice' can be created through building local support and creating demand for improved nutrition, and that lower levels of undernutrition can be achieved when this voice is used to increase political commitment, accountability and financing for nutrition ${ }^{(26)}$.

There is some evidence that participatory methodologies can also be a cost-effective and sustainable way to reduce child undernutrition. A randomised controlled trial in Nepal showed that participatory methodologies can harness the creativity, self-interest and self-organising activities of women who are poor to significantly reduce neonatal and maternal mortality through changes in home-care practices and health-care seeking ${ }^{(27)}$. A study in Kenya showed that multi-sectoral teams from relevant line ministries formed at district and divisional levels can significantly reduce child undernutrition by strengthening the capacity of people living in poor communities to have a voice in the decision-making processes and by increasing their access to basic government services ${ }^{(28)}$. However, an extensive review and analysis of experience concluded that to be successful these methodologies need to be embedded within community-driven programmes in an enabling environment provided by policies and interventions at all levels ${ }^{(29)}$.

\section{Raising woman's status in society}

There is strong evidence that increasing women's status can reduce child undernutrition. An analysis of demographic and health survey data in thirty-six countries (in South Asia, sub-Saharan Africa and Latin America and the Caribbean) found that increasing the status of women had a significant, positive effect on children's nutritional status in all three regions. The findings also showed that the very high rates of child undernutrition in South Asia, compared to sub-Saharan Africa, are associated with the much lower status of women in South Asia. The impact of women's lower status combines with the impact of poor sanitation and rapid urbanisation to make its impact on child undernutrition far higher in that region ${ }^{(30)}$. A more recent review of well-evaluated interventions that have credible evidence to show that within a short period of time women have become more empowered has identified four key policy options: (i) microfinance targeted at women; (ii) cash transfers targeted at women conditional on girls' attendance at school; (iii) reservation of positions for women in legislative bodies; and (iv) providing support for women's reproductive role ${ }^{(31)}$.

\section{Social protection policies}

Clearly, a comprehensive welfare state would solve the problem; but, once more, this is very unlikely in the short term. Proposed alternatives include targeted cash transfers. An analysis of twenty case studies in sub-Saharan Africa found that attempts to use cash transfers to strengthen social cohesion in poor communities have had varied results. They have sometimes proved to be socially divisive, leading to beneficiaries losing previous support from family or community, and have also fallen victim to 'elite capture'. However, there was some evidence that old-age pensions can increase the nutritional status of children ${ }^{(32)}$.

\section{Macro-economic policy reform}

In China, where dramatic reductions in child undernutrition have been achieved over the past two decades, macroeconomic policy reforms that have successfully stimulated economic growth, reduced poverty and addressed trade and agriculture have been associated with rapid improvements in child nutritional status ${ }^{(5)}$. These reforms have, however, been complemented by policies to support largescale implementation of effective nutrition, health, family planning, water and sanitation and education interventions $^{(6)}$. In Thailand, dramatic reductions in child undernutrition have been largely attributed to a supportive policy environment including a comprehensive nutrition policy, effective integration of nutrition within the National Economic and Social Development Plan and linkages between agriculture and nutrition for sustainability, together with successful community-level involvement and strong monitoring and evaluation ${ }^{(6)}$.

\section{Discussion}

The foregoing review has presented evidence for the effectiveness of a range of indirect interventions to address the drivers of child undernutrition, but for all these interventions context is all-important. Efforts should now be directed more towards improving implementation. 
The key challenges are how effective programmes can be implemented on a large scale in high-burden countries and how these programmes can be assessed at the macro (national and global), meso (local government/district) and micro (individual/family/household) levels.

\section{Key issues and challenges to implementation}

Large-scale implementation of effective interventions requires gaining high-level commitment to harness reliable, long-term funding and ensuring that funding of the technological fixes that directly improve nutritional status does not preclude funding of the indirect interventions that are crucial for sustainable improvement. It also requires increased recognition of the link between macroeconomic and social policies and child undernutrition; and the need to provide good management on the ground and effective use of information for awareness creation, monitoring, learning and evaluation. The challenges posed by these requirements are exacerbated by impediments to effective action such as HIV/AIDS and weak infrastructure.

There is a growing consensus that meeting these challenges will require a radical overhaul of the nutrition architecture. Any change needs to deliver stronger leadership, greater policy visibility, better coordination, clear messages, more effective technical support, significantly more financial resources and stronger linkages into country systems. A key challenge here is to achieve a more aggressive involvement of the nutrition community in finding new ways to work more effectively across sectors so that collaborative approaches can be designed.

Programme design and implementation also require choosing an appropriate entry point and making crosssectoral collaboration work. The choice of sector (e.g. agriculture, health, social development and local government) depends on which is most effective at the local level in a specific context. A key challenge here is to ensure that the interventions are culturally appropriate and cost-effective and that there are supportive national government policies and high-level political commitment to harness funds. Buy-in at the local level by community members is also needed for programme sustainability, along with the use of participatory methodologies and other strategies to increase demand-side factors to make governments more accountable for undernutrition.

The causes of undernutrition are known to be complex and to operate at different levels and across different sectors. They cannot, therefore, be addressed by any sector alone and multi-sectoral programmes are needed. Cross-sectoral collaboration has proved difficult in the past, but given the urgent need to take action, there is no choice but to document successes and try again. The foregoing review of evidence has shown that successful cross-sectoral interventions have been implemented by NGO and by multi-sectoral teams from relevant line ministries. The key challenge now is to build more multidisciplinary networks in high-burden countries and strengthen their capacity for nutrition advocacy and action. Incentives are also needed to encourage crosssectoral actions, such as community development funds dispersed through the district level that are ring-fenced for cross-sectoral actions.

\section{Key issues and challenges of assessment}

There is some evidence that community-driven programmes to raise women's status can be cost-effective, but there are few systematic costing data available and even less on costing community participation. There is notable variability with wide ranges in cost-benefit estimates. CCT programmes to increase uptake of $\mathrm{MCH}$ services are promising but have only been shown to reduce stunting in middle-income countries. They may also be cost-effective in low-income countries depending on the threshold value of the cash transfer needed to reduce child undernutrition and the cost of strengthening the health system to deliver adequate quality services. In the case of CCT to increase girls' attendance in school, additional costs may arise from the need to improve the quality of teaching and learning in the schools.

\section{Areas for furtber research and policy}

Attention needs to be focused on dissemination of existing evidence and experience and on methodology; there are also several areas where the answers are still unknown.

Dissemination is a neglected area. The wide range of studies and results cries out for more systematic dissemination of good practice. Country experiences of how to build commitment and develop and monitor multisectoral nutrition programmes need to be synthesised and disseminated. This should include experiences on how to move towards implementing cost-effective programmes on a large scale and how to reform or phase out ineffective programmes. There is also a need to better document successes (not just programme impact, but also impact pathways, using well-defined programme theory frameworks); and to develop a tool box of simple instruments to show policy makers the benefits of supporting nutrition from a political 'win-win' situation in regard to good governance, macro-economic/financial benefits and economic benefits from a livelihood/people perspective.

Issues of methodology are raised by variations in costing practices. There is a need for some degree of consensus on how to cost interventions where there is real community participation and on the procedures for making cost-effectiveness estimates. There is also a need to increase recognition that randomised controlled trials cannot be used to assess the importance of most of the underlying causes and some of the basic causes of child undernutrition, and to gain consensus on an attainable minimum of rigour. There is a need for greater clarity in research reports on the use of the term community involvement and for it to be related to a typology of 
participation because of the importance of factoring in community participation as a valued democratic outcome in itself. There is a need to further develop and assess valid indicators and methodologies that can be used at the national level and below to provide rapid feedback on progress in generating political commitment, strategic and operational capacities, coverage and effect.

Although the approaches briefly reviewed in the present paper have shown promise, they need to be confirmed/supported by further substantive research in areas where the answers are still unknown. Immediate suggestions are:

- What is the on-the-ground cost-effectiveness of biofortified crops produced and consumed by households? How can biofortified foods be made more acceptable to producers and consumers so that they increase their intake?

- What is the cost-effectiveness of CCT linked to the uptake of $\mathrm{MCH}$ services in improving child nutritional status in different low-income countries? How can the targeting of CCT avoid both 'elite capture' and further stigmatisation of the poorest and most vulnerable (including those affected by HIV)?

- What type of networks and other pathways support the translation of maternal education into improved child nutrition in different contexts?

- How can people's participation be assessed and ensured from the organisational and legal point of view, including ensuring access to data? What other strategies are effective in strengthening the demand side of governance at the local government level?

- What are the capacities, strategies and tactics present in countries that have been successful in advancing national nutrition agendas and actions, to guide national and international investments?

- How can cost-effective approaches to increasing women's status be implemented on a large scale? What approaches are effective for working with men on inequalities between men and women?

- What are the policy processes and the political and social conditions that contribute to improved child nutrition? What is the effect of changes in agricultural technologies and policy on nutritional outcomes in children? What are the linkages between nutrition outcomes and global change processes such as climate change, trade liberalisation, international migration and remittances, and long-term trends in fuel prices?

Equally, we have shown that reproducing effective programmes on a large scale is not easy. We need to know:

- What funding strategies can be used to encourage cross-sectoral programming? What multi-sectoral strategies can be used to effectively integrate long and short routes to improved child nutrition?
- How can capacity be built to deliver effective programmes on a large scale using different aid modalities/ public-private partnerships?

\section{Conclusion}

The problem of child undernutrition can be tackled through a range of effective interventions implemented on a large scale but the following preconditions need to be met for this to happen.

At the international (super-macro) level, donors need to keep to their commitments both in dollar terms and in terms of the 2005 Paris Declaration to ensure the most effective use of international aid through increased harmonisation, alignment and managing aid for results. However, given the changing nutrition architecture towards stronger leadership and greater policy visibility, it remains to be seen whether developing countries will seek ownership and put nutrition on their agenda so that donors can align behind these objectives and use local systems or whether the push for improved nutrition becomes donor driven.

At the national (macro) level, governance structures and institutions need to be in place to support an enabling environment for nutrition. Such an environment requires a culture of evidence-based decision making, accountability at all levels, inclusivity of private and voluntary sectors, promotion of equal rights for all and efforts to especially redress the inferior status of women in many societies.

At the meso (subnational) level, well-trained personnel need to be given the space to use their local knowledge in order to generate the best solutions for implementation, in exchange for being accountable to the local population. And at the micro (community) level, the chains of accountability have to be transparent; and the active involvement of households in decision-making processes is essential for the sustainability of any proposed intervention.

Child undernutrition in the 21st century contravenes human rights. It is simply embarrassing. The short-term solutions are known. Medium- to long-term solutions depend on our ability and willingness to collaborate and organise across sectors and across levels. Any further research has to be focused on implementation problems. Funding of interventions should choose from the above menus but with strong monitoring and evaluation components.

\section{Acknowledgements}

The present review was funded by the Danish Ministry of Foreign Affairs (Danida) who were not involved in finding or selecting the evaluations and studies reviewed, in the analysis, interpretation of data or writing of the 
report; or in the decision to submit the present paper for publication. The authors do not have any conflict of interests relating to the present review. P.P. and R.C.-H. collaborated in the selection and analysis of the evaluations and studies reviewed and in the writing of the report with P.P. taking a leading role.

\section{References}

1. World Health Organization (2008) Closing the Gap in a Generation: Health Equity Through Action on the Social Determinants of Health: Final Report of the Commission on Social Determinants of Health. Geneva: WHO.

2. Black RE, Allen LH, Bhutta ZA et al. (2008) Maternal and child undernutrition: global and regional exposures and health consequences. Lancet 371, 243-260.

3. Victora C, Adair L, Fall C et al. (2008) Maternal and child undernutrition: consequences for adult health and human capital. Lancet 371, 340-357.

4. Bhutta ZA, Ahmed T, Black RE et al. (2008) What works? Interventions for maternal and child undernutrition and survival. Lancet 371, 417-440.

5. Bryce J, Coitinho D, Darnton-Hill I et al. (2008) Maternal and child undernutrition: effective action at national level. Lancet 371, 510-526.

6. Ruel MT (2008) Addressing the underlying determinants of undernutrition: examples of successful integration of nutrition in poverty-reduction and agriculture strategies. UN ACC/SCN News 36, 18-21.

7. United Nations Children's Fund (1990) Strategy for Improved Nutrition of Children and Women in Developing Countries. A UNICEF Policy Review. New York: UNICEF.

8. Mosley WH \& Chen LC (1984) An analytic framework for the study of child survival in developing countries. Popul Dev Rev 10, 25-45.

9. Caldwell JC (1979) Education as a factor in mortality decline: an examination of Nigerian data. Popul Stud 33, $395-413$.

10. Hill K (2003) Frameworks for studying the determinants of child survival. Bull World Health Organ 81, 138-139.

11. McClafferty B \& Russell N (2002) Biofortification: Harnessing Agricultural Technology to Improve Health of the Poor. Washington, DC: IFPRI.

12. Nestel P, Bouis H, Meenakshi J et al. (2006) Biofortification of staple food crops. J Nutr 136, 1064-1067.

13. Potts MJ \& Naguija S (2007) A Review of Agriculture and Health Policies in Uganda with Implications for the Dissemination of Biofortified Crops. HarvestPlus Working Paper no. 1. Washington, DC: HarvestPlus.

14. Bhattacharjee L, Kumar SK \& Nandi BK (2007) Food-based Nutrition Strategies in Bangladesh: Experience of Integrated Horticulture and Nutrition Development. Bangkok: FAO, Regional Office for Asia and the Pacific.

15. Bushamuka VN, de Pee S, Talukder A et al. (2005) Impact of a homestead gardening program on household food security and empowerment of women in Bangladesh. Food Nutr Bull 26, 17-25.
16. World Bank and International Food Policy Research Institute (2007) From Agriculture to Nutrition: Pathways, Synergies and Outcomes. Washington, DC: World Bank.

17. Faber M, Phungula MAS, Venter S et al. (2002) Home gardens focusing on the production of yellow and darkgreen leafy vegetables increase the serum retinol concentrations of 2-5-y-old children in South Africa. Am J Clin Nutr 76, 1048-1054.

18. Frost MB, Forste R \& Haas DW (2005) Maternal education and child nutritional status in Bolivia: finding the links. Soc Sci Med 60, 395-407.

19. Walsh CM, Dannhauser A \& Joubert G (2002) The impact of a nutrition education programme on the anthropometric nutritional status of low-income children in South Africa. Public Health Nutr 5, 3-9.

20. Galasso E \& Umapathi N (2007) Improving Nutritional Status Through Behavioral Change: Lessons from Madagascar. Washington, DC: World Bank.

21. Sheikholeslam R, Kimiagar M, Siasi F et al. (2004) Multidisciplinary intervention for reducing malnutrition among children in the Islamic Republic of Iran. East Mediterr Health J 10, 844-852.

22. Lagarde M, Haines A \& Palmer N (2007) Conditional cash transfers for improving uptake of health interventions in low-and middle-income countries: a systematic review. JAMA 298, 1900-1910.

23. Gertler PJ (2004) Do conditional cash transfers improve child health? Evidence from Progresa's controlled randomized experiment. Am Econ Rev 94, 331-336.

24. Rivera JA, Sotres-Alvarez D, Habicht JP et al. (2004) Impact of the Mexican program for education, health, and nutrition (Progresa) on rates of growth and anemia in infants and young children: a randomized effectiveness study. JAMA 291, 2563-2570.

25. Sepulveda J, Bustreo F, Tapia R et al. (2007) Improvement of child survival in Mexico: the diagonal approach. Salud Publica Mex 49, S110-S125.

26. Rokx C (2006) Governance and Malnutrition: Exploring the Contribution of 'Good Governance' to Malnutrition Reduction in Developing Countries, in Faculty of Law. Enschede: University of Maastricht.

27. Manandhar D, Osrin D, Shrestha B et al. (2004) The effect of a participatory intervention with women's groups on birth outcomes in Nepal: cluster randomized controlled trial. Lancet 364, 970-979.

28. Havemann K (2005) Effective Participation for Health Development. London: Institute of Education, University of London.

29. Ismail S, Immink M, Mazar I et al. (2003) Community-based Food and Nutrition Programmes: What Makes Them Successful. Rome: FAO.

30. Smith LC, Ramakrishnan U Ndiaye A et al. (2003). The Importance of Women's Status for Child Nutrition in Developing Countries. Washington, DC: IFPRI.

31. King E, Klasen S \& Porter M (2008) Women and Development. Copenhagen: Copenhagen Consensus Centre.

32. Devereux S, Ellis F \& White P (2007) Regional Lesson Learning from the Case Studies, Case Study Briefs, Regional Evidence Building Agenda. Johannesburg: Wahenga, Regional Hunger and Vulnerability Programme (RHVP). 